\title{
Supersymmetric transformations for coupled channels with threshold differences
}

\author{
Boris F Samsonov $^{1}$, Jean-Marc Sparenberg ${ }^{2}$ and Daniel \\ Baye $^{2}$ \\ ${ }^{1}$ Physics Department, Tomsk State University, 36 Lenin Avenue, 634050 \\ Tomsk, Russia \\ 2 Physique Quantique, C P 229, Université Libre de Bruxelles, B 1050 Bruxelles, \\ Belgium \\ E-mail: samsonov@phys.tsu.ru, jmspar@ulb.ac.be and dbaye@ulb.ac.be
}

\begin{abstract}
The asymptotic behaviour of the superpotential of general SUSY transformations for a coupled channel Hamiltonian with different thresholds is analyzed. It is shown that asymptotically the superpotential can tend to a diagonal matrix with an arbitrary number of positive and negative entries depending on the choice of the factorization solution. The transformation of the Jost matrix is generalized to "non-conservative" SUSY transformations introduced in Sparenberg et al (2006 J. Phys. A: Math. Gen. 39 L639). Applied to the zero initial potential the method permits to construct superpartners with a nontrivially coupled Jost-matrix. Illustrations are given for two- and threechannel cases.

PACS numbers: 03.65.Nk,24.10.Eq
\end{abstract}

\section{Introduction}

In the context of quantum scattering inverse problems, not much is known about coupled-channel problems with threshold differences, i.e., inelastic problems (see paragraph IX.4 of [1] and references therein). Though well-known singlechannel methods based on the Marchenko or Gel'fand-Levitan approaches have been generalized to these coupled-channel problems, no sufficiently simple method for inverting experimental data has been deduced from these generalizations. This may be explained by the fact that in its general form the method requires knowing the whole set of scattering data and, in particular, the whole scattering $(S)$ matrix should be known, whereas only the open-channel submatrix is accessible from experimental data. Another reason is related with the complicated character of the Gel'fand-LevitanMarchenko equation which in this case was solved only numerically [2]. In the one channel case the second difficulty was overcome with the help of the supersymmetric quantum mechanics (SUSY QM) approach (see e.g. 3, 4]) which is equivalent to the Gel'fand-Levitan-Marchenko method when the kernel of the integral equation is degenerate [5, 6]. Moreover, when applied to the zero initial potential, these transformations lead to exactly-solvable potentials with scattering matrices being arbitrary-order rational functions of the wave number and hence providing excellent fits of experimental data [7]. Until recently, however, generalizing such supersymmetric 
transformations of the zero potential to inelastic coupled-channel problems seemed to be impossible as a matter of principle [8, 9, 10]. Thus, experimental data with coupling did not seem accessible to this method.

Fortunately, as it was recently announced [11, this strong limitation is explained by an unnecessary condition imposed on transformation functions used up to now. Usual supersymmetric transformations [3, 5, 6, 7, 8, 9, 10, can be described in terms of transformation operators relating solutions of two Sturm-Liouville problems, and hence keeping boundary conditions unchanged; this is why we call them "conservative". Changes in the spectrum due to such transformations may correspond only to elements from the kernel of either the transformation operator or its adjoint form. This is just the reason why the spectrum of two supersymmetric partners may differ only by a finite number of levels. Although the existence of transformations of another kind, breaking boundary conditions, is known for some time (see e.g. [12]), their use in the single-channel case has been limited to a very specific application [13, where usual transformations happen to be sufficient. In the inelastic coupledchannel case, on the contrary, these "non-conservative" transformations seem to be of fundamental importance as they lead to exactly-solvable potential models with nontrivially coupled $S$-matrices. We hope that these new transformations may become a keystone for an inversion procedure both simple enough for practical realization and accurate enough to fit experimental data with a good precision.

This renewal of interest for supersymmetric transformations of inelastic coupledchannel problems implies that a general study of their properties is necessary. In particular, the Jost-matrix transformation, from which the transformed S-matrix can be deduced, should be known in the general case. In [11, it is shown that the Jostmatrix modification depends on the value of the superpotential matrix $U(r)$ at infinity, which in turn depends on the asymptotic behaviour of the factorization solution. In the single-channel case, $U(\infty)$ is a positive (resp. negative) real number when the factorization solution increases (resp. decreases) at infinity. In the coupled-channel case, $U(r)$ is a matrix and its asymptotic behaviour is much more complicated. The main goal of the present work is to study carefully this behaviour.

Section 2 reviews definitions from multichannel scattering theory. As an introduction to Section 3 we first review briefly general properties of multichannel supersymmetric transformations and then we analyze the maximal number of arbitrary parameters entering into the superpotential and as a result into the transformed potential. Next we discuss in a detailed way the superpotential asymptotic behaviour proving our main theorem and give a closed expression for the transformed Jost solution and Jost function. In Section 4 we derive a general form of the superpotential which is a SUSY partner of the zero potential. Section 5 illustrates these findings by examples and comparisons with existing results from the literature. Section 6 contains conclusions and perspectives.

\section{Multichannel scattering}

Let us first define our notation and briefly recall some notions of scattering theory [14, 15] used below. We consider a multichannel radial Schrödinger equation that reads in reduced units

$$
H \psi(k, r)=k^{2} \psi(k, r) \quad H=-\frac{\mathrm{d}^{2}}{\mathrm{~d} r^{2}}+V
$$


where $r$ is the radial coordinate, $V$ is an $N \times N$ real symmetric matrix, and $\psi$ may be either a matrix-valued or a vector-valued solution. By $k$ we denote either a point in the space $\mathbb{C}^{N}, k=\left\{k_{1}, \ldots, k_{N}\right\}, k_{i} \in \mathbb{C}$ such that $\operatorname{Im} k_{i} \geqslant 0$, or a diagonal matrix with the non-vanishing entries $k_{i}, k=\operatorname{diag}\left(k_{1}, \ldots, k_{N}\right)$. The complex wave numbers $k_{i}$ are related to the center-of-mass energy $E$ and the channel thresholds $\Delta_{1}, \ldots, \Delta_{N}$, which are supposed to be different from each other, by

$$
k_{i}^{2}=E-\Delta_{i} .
$$

We do not assume a fixed order of channels since any necessary order can be achieved by a suitable permutation of the rows in the Schrödinger equation (11). For simplicity we will limit ourselves to unequal thresholds here and defer the general study of equal and unequal thresholds to a future work. We will also assume potential $V$ to be short-ranged at infinity, i.e., there exists an $\varepsilon>0$ such that

$$
\int_{0}^{\infty} \mathrm{e}^{\varepsilon r}\left|V_{i j}(r)\right| d r<\infty
$$

where $V_{i j}, i, j=1, \ldots, N$ are entries of matrix $V$. Under such assumptions, the Schrödinger equation has two $N \times N$ matrix-valued solutions $f( \pm k, r)$ (Jost solutions) such that

$$
f( \pm k, r) \underset{r \rightarrow \infty}{\rightarrow} \exp ( \pm \mathrm{i} k r)=\operatorname{diag}\left[\exp \left( \pm \mathrm{i} k_{1} r\right), \ldots, \exp \left( \pm \mathrm{i} k_{N} r\right)\right] .
$$

The columns of these matrices form a basis in the $2 N$-dimensional solution space of the Schrödinger equation with a given value of $E$. In general, these solutions are complex and satisfy the symmetry property $f(k, r)=f^{*}\left(-k^{*}, r\right)$, where asterisk denotes complex conjugation; for real energies below all thresholds, $k=-k^{*}$ and the Jost solutions are real.

Next we define the regular solution $\varphi(k, r)$ and the irregular solution $\eta(k, r)$ by their behaviour at the origin. For the sake of simplicity, we limit ourselves to bounded $s$-wave potentials, in which case these solutions satisfy

$$
\begin{array}{ll}
\varphi(k, 0)=0 & \varphi^{\prime}(k, 0)=I \\
\eta(k, 0)=I & \eta^{\prime}(k, 0)=0
\end{array}
$$

where prime means derivation with respect to $r$ and $I$ denotes the identity matrix. This definition shows that the columns of these matrices also form a basis in the solution space of the Schrödinger equation. In terms of the Jost solutions, these solutions read

$$
\begin{aligned}
& \varphi(k, r)=\frac{1}{2 \mathrm{i}}\left[f(k, r) k^{-1} F(-k)-f(-k, r) k^{-1} F(k)\right] \\
& \eta(k, r)=\frac{1}{2 \mathrm{i}}\left[f(k, r) k^{-1} G(-k)-f(-k, r) k^{-1} G(k)\right]
\end{aligned}
$$

where $F(k)$ is the Jost matrix

$$
F(k)=f^{T}(k, 0)
$$

with $T$ meaning transposition, and matrix $G(k)$ is defined as

$$
G(k)=-\left[f^{\prime}(k, 0)\right]^{T} .
$$

Proving (7) and (8) requires calculating, both at the origin and at infinity, the Wronskian $W[\varphi(k, r), f(k, r)] \equiv \varphi^{T}(k, r) f^{\prime}(k, r)-\left[\varphi^{\prime}(k, r)\right]^{T} f(k, r)$, which generalizes the usual definition of the Wronskian of one-component functions to $N$ channels, and the Wronskian $W[\eta(k, r), f(k, r)]$. Equation (11) implies that the value of these 
Wronskians is independent of $r$, as well as that of $W[f(-k, r), f(k, r)]=2 \mathrm{i} k$. For real energies, both solutions $\varphi$ and $\eta$ are purely real because they satisfy a system of differential equations with real coefficients and real boundary conditions. For energies below all thresholds or for energies above all thresholds, this can also be directly checked on (77) and (8), using the symmetry properties $F(k)=F^{*}\left(-k^{*}\right)$ and $G(k)=G^{*}\left(-k^{*}\right)$.

The Jost matrix defines both scattering and bound states properties. The scattering matrix, which is symmetric, reads

$$
S(k)=k^{-1 / 2} F(-k) F^{-1}(k) k^{1 / 2}=k^{1 / 2}\left[F^{-1}(k)\right]^{T} F^{T}(-k) k^{-1 / 2} .
$$

Bound-state energies, $E=E_{m}, m=1, \ldots, M$, correspond to zeros of the determinant of the Jost function, $\operatorname{det} F\left(\mathrm{i} \kappa_{m}\right) \equiv 0$, such that $\operatorname{Re} \kappa_{m, i}>0, \operatorname{Im} \kappa_{m, i}=0$ with $E_{m}=-\kappa_{m, i}^{2}+\Delta_{i}, i=1, \ldots, N$ below all thresholds. For potentials satisfying the above assumptions, the number $M$ of bound states is finite.

\section{Multichannel SUSY transformations}

According to the multichannel SUSY approach [8, 9, applying the transformation operator

$$
A^{-}=-\frac{\mathrm{d}}{\mathrm{d} r}+U(r)
$$

to solutions $\psi(k, r)$ of (1) leads to solutions $\tilde{\psi}(k, r)$ of the new equation

$$
\tilde{H} \tilde{\psi}(k, r)=k^{2} \tilde{\psi}(k, r) \quad \tilde{H}=-\frac{\mathrm{d}^{2}}{\mathrm{~d} r^{2}}+\tilde{V}(r)
$$

where $\tilde{V}$, like $V$, is supposed to be a real, short-ranged, bounded and symmetric $N \times N$ matrix and $\tilde{\psi}$, like $\psi$, may be either a matrix-valued or a vector-valued function. The matrix-valued function $U$ (usually called superpotential) is expressed in terms of a matrix-valued solution of (11) at a fixed value of $E=\mathcal{E}$ below all thresholds (this parameter is known as the factorization constant), which we denote $\sigma$ and call transformation function or factorization solution. Defining the corresponding wave number diagonal matrix $\kappa$ by its positive elements $\kappa_{i}=\sqrt{\Delta_{i}-\mathcal{E}}$, one has

$$
H \sigma(r)=-\kappa^{2} \sigma(r)
$$

and

$$
U(r)=\sigma^{\prime}(r) \sigma^{-1}(r)
$$

Then for $E \neq \mathcal{E}$ one has $\tilde{\psi}=A^{-} \psi$. The specific form (12), (15) of the transformation operator $A^{-}$results in the potential $\tilde{V}$ from (13) being of the form

$$
\tilde{V}(r)=V(r)-2 U^{\prime}(r) .
$$

To have a real and symmetric potential (16) we restrict $\sigma$ to be real and such that its self-Wronksian vanishes, $W(\sigma, \sigma)=0$.

For $E=\mathcal{E}$ a particular solution of (13) is

$$
\tilde{\phi}(\kappa, r)=\left(\sigma^{T}\right)^{-1}(r) \quad \tilde{H} \tilde{\phi}(\kappa, r)=-\kappa^{2} \tilde{\phi}(\kappa, r) .
$$

Other solutions $\tilde{\psi}$ corresponding to the same $\mathcal{E}$ may be found as usual from the property $W(\tilde{\phi}, \tilde{\psi})=-I$, which gives

$\tilde{\psi}(\kappa, r)=\left(\sigma^{T}\right)^{-1}(r) \int_{r_{0}}^{r} \sigma^{T}(s) \sigma(s) d s \quad \tilde{H} \tilde{\psi}(\kappa, r)=-\kappa^{2} \tilde{\psi}(\kappa, r)$. 
The conventional SUSY transformations have the property that if $\psi(0)=0$ then $\tilde{\psi}(0)=\left(A^{-} \psi\right)_{r=0}=0$ which requires some additional limitation on the transformation function $\sigma(r)$. Following [11] rejecting this limitation leads to loosing this property of the transformation operator $A^{-}$. Such transformation operators violate the vanishing behaviour of the solution at the origin and we call them "nonconservative". Nevertheless, since $A^{-}$transforms solutions of the initial differential equation into solutions of the transformed equation the full information about the new Hamiltonian $\tilde{H}$ is accessible. In particular its Jost function and $S$-matrix can be constructed explicitly. Below we will concentrate our attention mainly on nonconservative transformations although our main result (Theorem 2 in Section 3.2) is valid for the general case.

\subsection{Number of arbitrary parameters in superpotential}

First we discuss the general form of the transformation function $\sigma$ introduced in the previous section. We notice that the general vector-valued solution of the Schrödinger equation (10) contains $2 N$ integration constants and can always be presented as a linear combination of $2 N$ fixed linearly independent solutions. In general, transformation function $\sigma$ may be composed of $N$ such solutions. Therefore it may contain $2 N^{2}$ arbitrary parameters at most. But as far as the new potential (16) is concerned there is a big redundancy between these parameters. Indeed, because of the specific form of the superpotential (15) a multiplication of the transformation function $\sigma$ on the right by a non-singular constant matrix does not affect the superpotential. A minimal set of arbitrary parameters is given by

Theorem 1. Given the initial potential $V(r)$, fixed thresholds and factorization energy, the most general transformed potential $\tilde{V}(r)$ is completely determined by the value $U(0)$ of the symmetric superpotential matrix at the origin. It is calculated by formulas (16) and (15) where

$$
\sigma(r)=\eta(\mathrm{i} \kappa, r)+\varphi(\mathrm{i} \kappa, r) U(0)
$$

and contains $N(N+1) / 2$ arbitrary real parameters which are the entries of matrix $U(0)$.

Proof. The general matrix-valued solution of the Schrödinger equation

$$
\sigma(r)=\eta(\mathrm{i} \kappa, r) C_{1}+\varphi(\mathrm{i} \kappa, r) D_{1},
$$

with real matrices $C_{1}$ and $D_{1}$ produces the most general real superpotential (15) and, hence, potential (16). Definitions (5) and (6) then imply that $\operatorname{det} \sigma(0)=\operatorname{det} C_{1}$ vanishes if and only if matrix $C_{1}$ is singular. In this case $\sigma(0)$ is not invertible and the superpotential and hence the transformed potential $\tilde{V}$ become singular at the origin; as stated above, we want to avoid this case here and therefore we impose the condition $\operatorname{det} C_{1} \neq 0$. It is now clear that in this case matrix $C_{1}$ does not affect the superpotential $U$ as given in (15) since we can multiply (20) by $C_{1}^{-1}$ on the right, which leaves the superpotential unaffected or, equivalently without loosing generality, put $C_{1}=I$. The superpotential $U$ and hence the transformed potential $\tilde{V}$ thus only depend on the $N^{2}$ parameters appearing in $D_{1}$.

This simplified writing allows us to express easily the other condition imposed on the real transformation function (20), namely the symmetry of $U$ and $\tilde{V}$. As mentioned above, this happens when $W(\sigma, \sigma)=0$, which gives $N(N-1) / 2$ equations 
for the elements of matrix $D_{1}$. The value of this Wronskian being $r$-independent, (20) can be used to calculate it at the origin with (5) and (6) which leads to

$$
W[\sigma(r), \sigma(r)]=D_{1}-D_{1}^{T}=0 .
$$

The superpotential and transformed potential are thus symmetric when $D_{1}$ is chosen symmetric. This can also be checked on the value of the superpotential at the origin which reads, according to (5), (6), (15) and (20), $U(0)=D_{1}$.

To calculate the Jost matrix for the transformed potential according to (9) we need to know its Jost solution which is defined by the asymptotic behaviour (44). Usually a supersymmetry transformation changes this behaviour. So we have to analyze the asymptotic behaviour of the function $\tilde{f}(k, r)=A^{-} f(k, r)$ which is mainly defined by the asymptotics of superpotential $U(r)$.

\subsection{Asymptotic behaviour of superpotential}

According to (15), the asymptotic behaviour of the superpotential depends on the asymptotic behaviour of the factorization solution. This time, in place of (20), we choose to write the factorization solution as

$$
\sigma(r)=f(-\mathrm{i} \kappa, r) \kappa^{-1 / 2} C_{2}+f(\mathrm{i} \kappa, r) \kappa^{-1 / 2} D_{2}
$$

with $C_{2}$ and $D_{2}$ being some constant matrices; factor $\kappa^{-1 / 2}$ is introduced for further convenience. We notice that matrices $C_{2}$ and $D_{2}$ should satisfy the condition

$$
D_{2}^{T} C_{2}-C_{2}^{T} D_{2}=0
$$

following from the symmetry property of the superpotential $W[\sigma(r), \sigma(r)]=0$.

We show below that the asymptotic behaviour of the superpotential crucially depends on the structure of matrix $C_{2}$. In particular, it depends on the rank of $C_{2}$ and if $\operatorname{rank} C_{2}=R<N$ it depends on an interrelation between the values of thresholds and linear dependence between rows of $C_{2}$. This interrelation becomes more transparent for a specific order of channels. Therefore before going further we will first rearrange the channels taking into account the structure of matrix $C_{2}$. Our main aim in this reordering is to collect together both all linearly independent rows of matrix $C_{2}$ and its linearly independent columns. As it was already noticed changing channels corresponds to going to another starting Hamiltonian (11). But evidently it corresponds to the same physical system after the reordering. The permutation of columns simultaneously both in $C_{2}$ and in $D_{2}$ is equivalent to a multiplication on the right of the whole factorization solution $\sigma$ by a constant non-singular matrix which evidently does not change the superpotential $U$ as given by (15).

We rearrange the rows of $C_{2}$ together with the corresponding channels in the following way. The first channel with wavenumber $\kappa_{1}^{\prime}$ and, hence, the first row of the reordered matrix (we denote $C_{3}$ ) correspond to the largest threshold related to a non-vanishing row of $C_{2}$. The second channel with wavenumber $\kappa_{2}^{\prime}$ and, hence, the second row of the new matrix $C_{3}$ correspond to the largest remaining threshold related to a row of $C_{2}$ linearly independent of the first row of $C_{2}$. At each next step $i \leq R$, a new channel with wavenumber $\kappa_{i}^{\prime}$ and row $i$ of $C_{3}$ corresponds to the largest remaining threshold related to a row of $C_{2}$ linearly independent of the previous rows of $C_{3}$. The reordering ends when the first $R$ rows of $C_{3}$ become linearly independent. All remaining rows of $C_{2}$ are transferred to $C_{3}$ without changes. As mentioned above 
we now permute columns in $C_{3}$ to have its upper left $R \times R$ block non-singular thus obtaining matrix $C$. Matrix $D_{2}$ after all these permutations is transformed into $D$.

The diagonal wavenumber matrix is written as

$$
\kappa=\left(\begin{array}{cc}
\kappa^{\prime} & 0 \\
0 & \kappa^{\prime \prime}
\end{array}\right)
$$

where $\kappa^{\prime}$ is the $R \times R$ diagonal block after the reordering and $\kappa^{\prime \prime}$ is the $(N-R) \times(N-R)$ diagonal block containing the remaining wavenumbers. Such a structure of $C, D$ and wavenumber matrices will be assumed till the end of the paper.

Now we can formulate our main theorem.

Theorem 2. When $r \rightarrow \infty$, if rank $C=R$, the superpotential has the asymptotic form

$$
\begin{aligned}
& U(r) \rightarrow \operatorname{diag}\left(u_{i i}\right) \\
& u_{i i}=+\kappa_{i} \quad i=1, \ldots, R \\
& u_{i i}=-\kappa_{i} \quad i=R+1, \ldots, N .
\end{aligned}
$$

In order to prove the theorem, we first reduce $C$ and $D$ to canonical forms, simplest as far as the superpotential (15) is concerned but reflecting on the one hand the singular character of matrix $C$ and on the other hand the non-singularity of the whole factorization solution $\sigma(r)$. This is performed in the next lemma.

Lemma 1. Matrices $C$ and $D$ can be transformed by right multiplication with a nonsingular square matrix $T$ into the canonical forms

$$
C T=\left(\begin{array}{cc}
I & 0 \\
Q_{0} & 0
\end{array}\right) \quad D T=\left(\begin{array}{cc}
X_{0} & -Q_{0}^{T} \\
0 & I
\end{array}\right)
$$

where $I$ denotes the $R \times R$ unit matrix in $C T$ and the $(N-R) \times(N-R)$ unit matrix in DT and $X_{0}$ is a symmetric matrix, $X_{0}^{T}=X_{0}$. Matrix $Q_{0}$ verifies the following property. For any $i \leq R$ and $j>R$ such that the inequality $\kappa_{i}^{\prime}<\kappa_{j}^{\prime \prime}$ holds one has

$$
q_{j i}^{0}=0
$$

where $q_{j i}^{0}$ are entries of matrix $Q_{0}$.

Proof. By construction the $R \times R$ upper left block $M$ of $C$ is invertible. Since the rank of $C$ is $R$, the last $N-R$ columns of $C$ are linear combinations of the first $R$ ones. This means that there exists an $R \times(N-R)$ matrix $P$ such that

$$
C\left(\begin{array}{c}
P \\
-I
\end{array}\right)=0
$$

where $I$ is the $(N-R) \times(N-R)$ unit matrix. Similarly, for the rows, there exists an $(N-R) \times R$ matrix $Q_{0}$ such that

$$
\left(\begin{array}{cc}
Q_{0} & -I
\end{array}\right) C=0 .
$$

Hence matrix $C$ can be written as

$$
C=\left(\begin{array}{cc}
M & M P \\
Q_{0} M & Q_{0} M P
\end{array}\right)
$$

or

$$
C=\left(\begin{array}{cc}
I & 0 \\
Q_{0} & 0
\end{array}\right)\left(\begin{array}{cc}
M & 0 \\
0 & I
\end{array}\right)\left(\begin{array}{cc}
I & P \\
0 & -I
\end{array}\right)
$$


and

$$
C T_{1}=\left(\begin{array}{cc}
I & 0 \\
Q_{0} & 0
\end{array}\right) \quad T_{1}=\left(\begin{array}{cc}
I & P \\
0 & -I
\end{array}\right)\left(\begin{array}{cc}
M & 0 \\
0 & I
\end{array}\right)^{-1}
$$

The linear dependence of the rows is not modified by the multiplication of $C$ by a nonsingular $T_{1}$ after which row $i(\leq R)$ contains only one non-vanishing element in column $i$. Property (29) now follows from the fact that by construction row $j$ is a linear combination of all previous rows $i=1,2, \ldots$ of $C$ such that $\kappa_{i}^{\prime}>\kappa_{j}^{\prime \prime}$.

Instead of matrix $D_{2}$ the product

$$
D T_{1}=\left(\begin{array}{cc}
D_{11} & D_{12} \\
D_{21} & D_{22}
\end{array}\right)
$$

now appears in (22). According to (23) the symmetry property of the superpotential is translated into the following conditions:

$$
\begin{aligned}
& D_{11}-D_{11}^{T}=D_{21}^{T} Q_{0}-Q_{0}^{T} D_{21} \\
& D_{12}=-Q_{0}^{T} D_{22} .
\end{aligned}
$$

Equation (37) together with form (34) of $C T_{1}$ implies that if $D_{22}$ in (35) is singular, function $\sigma(r)$ given in (22) becomes singular for all $r$, a case we would like to avoid here so that we necessarily assume $D_{22}$ to be invertible. Moreover, using (36) and (37) we rewrite matrix $D T_{1}$ as

$$
D T_{1}=\left(\begin{array}{cc}
X_{0} & -Q_{0}^{T} \\
0 & I
\end{array}\right)\left(\begin{array}{cc}
I & 0 \\
D_{21} & D_{22}
\end{array}\right)
$$

where we denoted $X_{0}=D_{11}+Q_{0}^{T} D_{21}$. Finally, we can define $T=T_{1} T_{2}$ where $T_{1}$ is given by (34) and $T_{2}$ is the inverted second factor in the right hand side of (38) which keeps unchanged matrix $C T_{1}$; the condition $X_{0}^{T}=X_{0}$ follows from (36).

In the next lemma we establish an important property of matrix-valued function $Q(r)$ which will appear in the asymptotic form of the factorization solution.

Lemma 2. For $\kappa^{\prime}, \kappa^{\prime \prime}$ and $Q_{0}$ defined as in Lemma 1, the $(N-R) \times R$ matrix

$$
Q(r)=\mathrm{e}^{\kappa^{\prime \prime} r} Q_{0} \mathrm{e}^{-\kappa^{\prime} r}
$$

tends to zero when $r$ tends to infinity.

Proof. A matrix element of $Q$ is given by

$$
q_{j i}(r)=q_{j i}^{0} \mathrm{e}^{\left(\kappa_{j}^{\prime \prime}-\kappa_{i}^{\prime}\right) r}
$$

From Lemma 1, either the exponential tends to zero or coefficient $q_{j i}^{0}$ vanishes.

Now we can prove Theorem 2 .

Proof. According to (4), the factorization solution has the following asymptotic behaviour

$$
\sigma(r) \underset{r \rightarrow \infty}{\rightarrow} \sigma_{\text {as }}(r)=\kappa^{-1 / 2}\left[\mathrm{e}^{\kappa r} C+\mathrm{e}^{-\kappa r} D\right] .
$$

Recalling that $C$ and $D$ here have canonical forms (28) we get

$$
\sigma_{\text {as }}(r)=\kappa^{-1 / 2}\left(\begin{array}{cc}
I+X & -Q^{T} \\
Q & I
\end{array}\right)\left(\begin{array}{cc}
\mathrm{e}^{\kappa^{\prime} r} & 0 \\
0 & \mathrm{e}^{-\kappa^{\prime \prime} r}
\end{array}\right)
$$


where $Q$ is given by (39) and matrix

$$
X(r)=\mathrm{e}^{-\kappa^{\prime} r} X_{0} \mathrm{e}^{-\kappa^{\prime} r}
$$

vanishes at infinity. The derivative of (42) can be written as

$$
\sigma_{\text {as }}^{\prime}(r)=\kappa^{1 / 2}\left(\begin{array}{cc}
I-X & Q^{T} \\
Q & -I
\end{array}\right)\left(\begin{array}{cc}
\mathrm{e}^{\kappa^{\prime} r} & 0 \\
0 & \mathrm{e}^{-\kappa^{\prime \prime} r}
\end{array}\right)
$$

Hence, for $U$ one has the following asymptotic behaviour

$U(r) \underset{r \rightarrow \infty}{\rightarrow} U_{\mathrm{as}}(r)=\kappa^{1 / 2}\left(\begin{array}{cc}I-X & Q^{T} \\ Q & -I\end{array}\right)\left(\begin{array}{cc}I+X & -Q^{T} \\ Q & I\end{array}\right)^{-1} \kappa^{1 / 2}$

From Lemma 2 and (43) one obtains for $r \rightarrow \infty$

$$
U_{\text {as }}(r) \rightarrow \kappa\left(\begin{array}{cc}
I & 0 \\
0 & -I
\end{array}\right),
$$

which concludes the proof.

Corollary 1. If the thresholds are ordered such that $\kappa_{1}>\kappa_{2}>\ldots>\kappa_{N}$ and matrix $C$ has rank $R$ the number of arbitrary parameters in the superpotential is $R(R+1) / 2+R(N-R)$.

Proof. For the given order of thresholds all $q_{j i}^{0}$ may be chosen different of zero according to Lemma 1. Moreover, the parameters enter in the superpotential only through matrices $X_{0}$ and $Q_{0}$.

\subsection{Jost-matrix transformation}

After the asymptotics of the superpotential is found we are able to calculate both the Jost solution and the Jost matrix for the transformed potential.

Theorem 3. The Jost matrix $\tilde{F}(k)$ of the transformed potential $\tilde{V}$ reads, in terms of the Jost matrix $F(k)$ and the function $G(k)$ (10) of the initial potential,

$$
\tilde{F}(k)=[U(\infty)-\mathrm{i} k]^{-1}[F(k) U(0)+G(k)] .
$$

Proof. According to Theorem 2 the Jost solution of the transformed potential reads

$$
\tilde{f}(k, r)=A^{-} f(k, r)[U(\infty)-\mathrm{i} k]^{-1}
$$

as seen with (12) and (4). The theorem then follows from definitions (9) and (10). 


\section{SUSY partners of $V(r) \equiv 0$}

The zero initial potential is important since in this case compact analytic expressions are possible both for the transformed potential and for its Jost function. The initial Jost solution in this case is simply the exponential $f(k, r)=\exp (\mathrm{i} k r)$ and the initial Jost function is the identity matrix, $F(k)=I$. Hence, $G(k)=-\mathrm{i} k$. The regular solution has the form: $\varphi(k, r)=\sin (k r) k^{-1}$ and the irregular solution is written as $\eta(k, r)=\cos (k r)$. The factorization solution respecting Theorem 1 has the form

$$
\begin{aligned}
\sigma(r) & =\cosh (\kappa r)+\sinh (\kappa r) \kappa^{-1} U(0) \\
& =\frac{1}{2} \mathrm{e}^{\kappa r}\left[I+\kappa^{-1} U(0)\right]+\frac{1}{2} \mathrm{e}^{-\kappa r}\left[I-\kappa^{-1} U(0)\right] .
\end{aligned}
$$

Another parametrization corresponds to Lemma 1

$$
\sigma(r)=\kappa^{-1 / 2}\left[\mathrm{e}^{\kappa r} C+\mathrm{e}^{-\kappa r} D\right]
$$

with an appropriate choice of matrices $C$ and $D$.

Proposition 1. Let $I$ be the $R \times R$ identity matrix, matrices $X_{0}$ and $Q_{0}$ be chosen according to Lemma 1, $Q(r)$ be defined by (39) and $X(r)$ by (43). If the parameters are such that $\operatorname{det} Y(r) \neq 0 \forall r \in[0, \infty)$ where

$$
Y(r)=I+X(r)+Q^{T}(r) Q(r)
$$

then the potential $\tilde{V}(r)=-2 U^{\prime}(r)$ with

$$
U=-\kappa+2 \kappa^{1 / 2}\left(\begin{array}{cc}
Y^{-1} & Y^{-1} Q^{T} \\
Q Y^{-1} & Q Y^{-1} Q^{T}
\end{array}\right) \kappa^{1 / 2}
$$

is a (non-conservative) SUSY partner of $V(r) \equiv 0$ and has the Jost solution

$$
\tilde{f}(k, r)=[U(r)-\mathrm{i} k] \mathrm{e}^{\mathrm{i} k r}[U(\infty)-\mathrm{i} k]^{-1}
$$

and the Jost function

$$
\tilde{F}(k)=[U(\infty)-\mathrm{i} k]^{-1}[U(0)-\mathrm{i} k]
$$

where $U(\infty)$ may be found from (25,27).

Proof. First we notice that the function $\sigma(r)=\sigma_{\text {as }}(r)$ given in (41) is just function (51) and, hence, it can be taken as transformation function to produce a SUSY partner of $V(r) \equiv 0$. For $C$ and $D$ in block forms (28) the superpotential is given in (45). From here after some algebra one gets (53). Expressions (54) and (55) for the Jost solution and the Jost function correspond to (48) and (47) for the zero initial potential, respectively.

Corollary 2. For $X_{0}=0$ the superpotential (53) can be written as

$$
\begin{aligned}
U & =\kappa-2 \kappa^{1 / 2} Q_{r}^{T}\left(Q_{r} Q_{r}^{T}\right)^{-1} Q_{r} \kappa^{1 / 2} \\
& =-\kappa+2 \kappa^{1 / 2} Q_{c}\left(Q_{c}^{T} Q_{c}\right)^{-1} Q_{c}^{T} \kappa^{1 / 2}
\end{aligned}
$$

where $Q_{r}$ and $Q_{c}$ are row and column block matrices written in terms of $Q$ (39) as $Q_{r}=(Q,-I)$ where $I$ is the $(N-R) \times(N-R)$ identity matrix, $Q_{c}^{T}=\left(I, Q^{T}\right)$ where $I$ is the $R \times R$ identity matrix. 
Proof. Using the property

$$
Q\left[I+Q^{T} Q\right]^{-1}=\left[I+Q Q^{T}\right]^{-1} Q
$$

one obtains from (53)

$$
\begin{aligned}
U & =-\kappa+2 \kappa^{1 / 2}\left(\begin{array}{cc}
I & Q^{T} \\
Q & Q Q^{T}
\end{array}\right)\left(\begin{array}{cc}
I+Q^{T} Q & 0 \\
0 & I+Q Q^{T}
\end{array}\right)^{-1} \kappa^{1 / 2} \\
& =\kappa-2 \kappa^{1 / 2}\left(\begin{array}{cc}
Q^{T} Q & -Q^{T} \\
-Q & I
\end{array}\right)\left(\begin{array}{cc}
I+Q^{T} Q & 0 \\
0 & I+Q Q^{T}
\end{array}\right)^{-1} \kappa^{1 / 2} .
\end{aligned}
$$

Equations (56) and (57) are nothing but compact forms of (60) and (59) respectively.

For two particular cases corresponding to rank $C=N-1$ and rank $C=1$ either (56) or (57) takes a particularly simple form since in one case $Q_{r}$ is a row and in the other case $Q_{c}$ is a column. The explicit expressions are given in the following corollary:

Corollary 3. Let channels be ordered such that $\kappa_{1}>\kappa_{2}>\ldots>\kappa_{N-1}$. Let also $Q$ be a row, $Q=\left(q_{1}, \ldots, q_{N-1}\right), q_{i}=q_{i}^{0} \exp \left(\kappa_{N}-\kappa_{i}\right) r$ where $q_{i}^{0}=0$ for any $i$ such that $\kappa_{N}>\kappa_{i}$ and arbitrary otherwise. Then the superpotential has the following block form:

$$
U=\kappa-\frac{2}{1+Q Q^{T}} \kappa^{1 / 2}\left(\begin{array}{cc}
Q^{T} Q & -Q^{T} \\
-Q & 1
\end{array}\right) \kappa^{1 / 2}
$$

where $Q^{T} Q$ is an $(N-1) \times(N-1)$ matrix with entries $q_{i} q_{j}, i, j=1, \ldots, N-1$.

Let us now order only the first channel such that $\kappa_{1}>\max \left(\kappa_{2}, \ldots, \kappa_{N}\right)$ and $Q^{T}$ be a row $Q^{T}=\left(q_{2}, \ldots, q_{N}\right), q_{i}=q_{i}^{0} \exp \left(\kappa_{i}-\kappa_{1}\right) r$. Then the superpotential may be written as

$$
U=-\kappa+\frac{2}{1+Q^{T} Q} \kappa^{1 / 2}\left(\begin{array}{cc}
1 & Q^{T} \\
Q & Q Q^{T}
\end{array}\right) \kappa^{1 / 2}
$$

where $Q Q^{T}$ is an $(N-1) \times(N-1)$ matrix with entries $q_{i} q_{j}, i, j=2, \ldots, N$.

Proof. The statement follows from Lemma 1 and Corollary 2. For the first part of the statement rank $C=N-1$ whereas for the second part rank $C=1$.

Another simplification occurs for rank $C=N$ and a particular choice of matrix $X_{0}$.

Corollary 4. Let $\mathcal{X}_{0}$ be a column of $N$ arbitrary real parameters and $\mathcal{X}=$ $\exp (-\kappa r) \mathcal{X}_{0}$. Then the superpotential reads

$$
U=\kappa-\frac{2}{1+\mathcal{X}^{T} \mathcal{X}} \kappa^{1 / 2} \mathcal{X} \mathcal{X}^{T} \kappa^{1 / 2} .
$$

Proof. Choosing rank $C=N$ (meaning that $Q_{0}=0$ ) and $X_{0}=\mathcal{X}_{0} \mathcal{X}_{0}^{T}$, we have $Y=I+\mathcal{X X}^{T}$. Using the property $\left(\mathcal{X X}^{T}\right)^{2}=\left(\mathcal{X}^{T} \mathcal{X}\right) \mathcal{X} \mathcal{X}^{T}$ one gets $Y^{-1}=$ $I-\left(1+\mathcal{X}^{T} \mathcal{X}\right)^{-1} \mathcal{X} \mathcal{X}^{T}$. The statement follows now from (53).

\section{Examples}

Let us now illustrate the theorems and Proposition 1 we have just established by some exactly-solvable examples, supersymmetric partners of $V(r) \equiv 0$. 


\subsection{The $2 \times 2$ model with rank $C=2$ (Cox potential)}

Let us start from a two-channel problem.

According to Theorem 10 we choose the transformation function as given in (50) with the maximal number of arbitrary parameters included in

$$
U(0)=\left(\begin{array}{cc}
\alpha_{1} & \beta \\
\beta & \alpha_{2}
\end{array}\right) .
$$

Taking (50) into account, the condition rank $C=2$ reads

$$
\left(\kappa_{1}+\alpha_{1}\right)\left(\kappa_{2}+\alpha_{2}\right)-\beta^{2} \neq 0 .
$$

Because of the simple character of the transformation function we easily find the superpotential according to (15). Its off diagonal elements have the form: $u_{12}=$ $u_{21}=\beta / \operatorname{det} \sigma$. For the first diagonal element one obtains

$$
\begin{aligned}
& u_{11}=\frac{\cosh \left(\kappa_{2} r\right)}{\operatorname{det} \sigma}\left[\alpha_{1} \cosh \left(\kappa_{1} r\right)+\kappa_{1} \sinh \left(\kappa_{1} r\right)\right] \\
& +\frac{\sinh \left(\kappa_{2} r\right)}{\kappa_{2} \operatorname{det} \sigma}\left[\left(\alpha_{1} \alpha_{2}-\beta^{2}\right) \cosh \left(\kappa_{1} r\right)+\kappa_{1} \alpha_{2} \sinh \left(\kappa_{1} r\right)\right] .
\end{aligned}
$$

Here

$$
\begin{aligned}
& \operatorname{det} \sigma=\frac{\alpha_{2}}{\kappa_{2}} \cosh \left(\kappa_{1} r\right) \sinh \left(\kappa_{2} r\right)+\frac{\alpha_{1}}{\kappa_{1}} \cosh \left(\kappa_{2} r\right) \sinh \left(\kappa_{1} r\right) \\
& +\cosh \left(\kappa_{1} r\right) \cosh \left(\kappa_{2} r\right)+\frac{\alpha_{1} \alpha_{2}-\beta^{2}}{\kappa_{1} \kappa_{2}} \sinh \left(\kappa_{1} r\right) \sinh \left(\kappa_{2} r\right) .
\end{aligned}
$$

The element $u_{22}$ is obtained from (66) by the replacement $\kappa_{1} \leftrightarrow \kappa_{2}$ and $\alpha_{1} \leftrightarrow \alpha_{2}$. According to (16) the transformed potential is simply twice the derivative of these expressions with the opposite sign.

Since rank $C=2$, Theorem 2 states that no negative entries in the asymptotic form of the superpotential can appear so that $U(\infty)=\kappa=\operatorname{diag}\left(\kappa_{1}, \kappa_{2}\right)$ which can

also be checked by a direct calculation. Applying now Theorem 3 we find the Jost matrix for this potential

$$
\tilde{F}(k)=\left(\begin{array}{cc}
\frac{\alpha_{1}-\mathrm{i} k_{1}}{\kappa_{1}-\mathrm{i} k_{1}} & \frac{\beta}{\kappa_{1}-\mathrm{i} k_{1}} \\
\frac{\beta}{\kappa_{2}-\mathrm{i} k_{2}} & \frac{\alpha_{2}-\mathrm{k} k_{2}}{\kappa_{2}-\mathrm{i} k_{2}}
\end{array}\right)
$$

We notice that up to a change of parameters we obtain one of the two-channel Bargmann potentials previously found by other means [16].

The same superpotential may be rewritten with another parametrization. According to Proposition 1 for $\operatorname{rank} C=N$ the superpotential reads $U=-\kappa+$ $2 \kappa^{1 / 2}(I+X)^{-1} \kappa^{1 / 2}$ with $X$ given by (43) the elements of which are $x_{i j}=\exp \left(-\kappa_{i} r-\right.$ $\left.\kappa_{j} r\right) x_{i j}^{0}, i, j=1,2$. Its more explicit form is

$U=-\kappa+\frac{2}{\left(1+x_{11}\right)\left(1+x_{22}\right)-x_{12}^{2}} \kappa^{1 / 2}\left(\begin{array}{cc}1+x_{22} & -x_{12} \\ -x_{12} & 1+x_{11}\end{array}\right) \kappa^{1 / 2}$.

After the change of the parameters

$$
\begin{aligned}
& x_{11}^{0}=\frac{1}{\operatorname{det}[U(0)+\kappa]}\left[\beta^{2}-\left(\alpha_{1}-\kappa_{1}\right)\left(\alpha_{2}+\kappa_{2}\right)\right] \\
& x_{22}^{0}=\frac{1}{\operatorname{det}[U(0)+\kappa]}\left[\beta^{2}-\left(\alpha_{1}+\kappa_{1}\right)\left(\alpha_{2}-\kappa_{2}\right)\right] \\
& x_{12}^{0}=\frac{-2 \beta \sqrt{\kappa_{1} \kappa_{2}}}{\operatorname{det}[U(0)+\kappa]}
\end{aligned}
$$



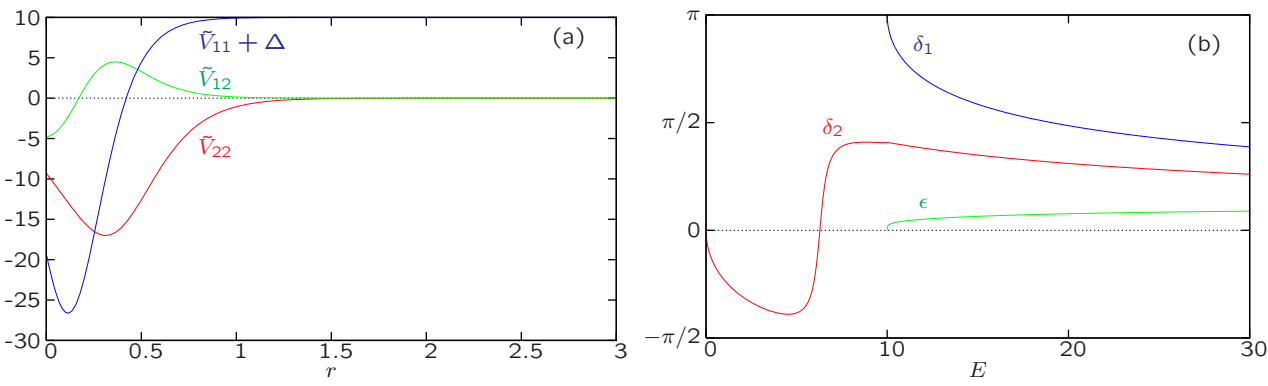

Figure 1. $2 \times 2$ exactly-solvable potential (a) and scattering matrix (b) for the choice of parameters (73)-75) and $\kappa_{2}=3($ rank $C=2)$.
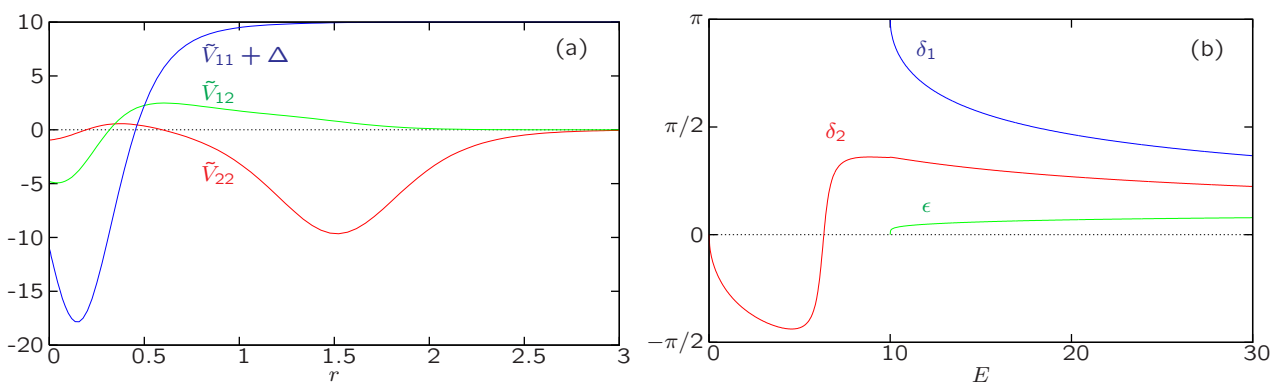

Figure 2. Same as figure 1 but for $\kappa_{2}=2.2(\operatorname{rank} C=2)$.

one recovers the previous result.

Two explicit examples of this $2 \times 2$ model are given in figures 1 and 2, Part (a) of each figure shows the potential while part (b) shows the eigenphase shifts $\delta_{1}, \delta_{2}$ and the mixing parameter $\epsilon$ [15]. For these examples, we have chosen the thresholds

$$
\Delta_{1}=\Delta=10, \quad \Delta_{2}=0,
$$

which implies that the factorization energy $\mathcal{E}$ and wave number $\left\{\kappa_{1}, \kappa_{2}\right\}$ are related by

$$
\kappa_{1}^{2}=\Delta-\mathcal{E}>\kappa_{2}^{2}=-\mathcal{E}
$$

In both cases, the value of the superpotential at the origin, (64), is chosen as

$$
U(0)=\left(\begin{array}{cc}
-2 & 0.6 \\
0.6 & -2
\end{array}\right)
$$

while the factorization wave number is $\kappa_{2}=3$ and $\kappa_{2}=2.2$ in figures 1 and 2 respectively. Comparison of figures 1 (a) and 2 (a) shows that the choice of factorization energy strongly modifies the potential. In contrast, figures 1 (b) and 2 (b) show that this choice does not strongly affect the scattering matrix, which always displays a Feshbach resonance at an energy of about 6.3 and a large negative slope of $\delta_{2}$ at zero energy and of $\delta_{1}$ above threshold. The eigenphase shifts and mixing parameter are only a bit smaller in figure 2 (b) than in figure 1(b). 

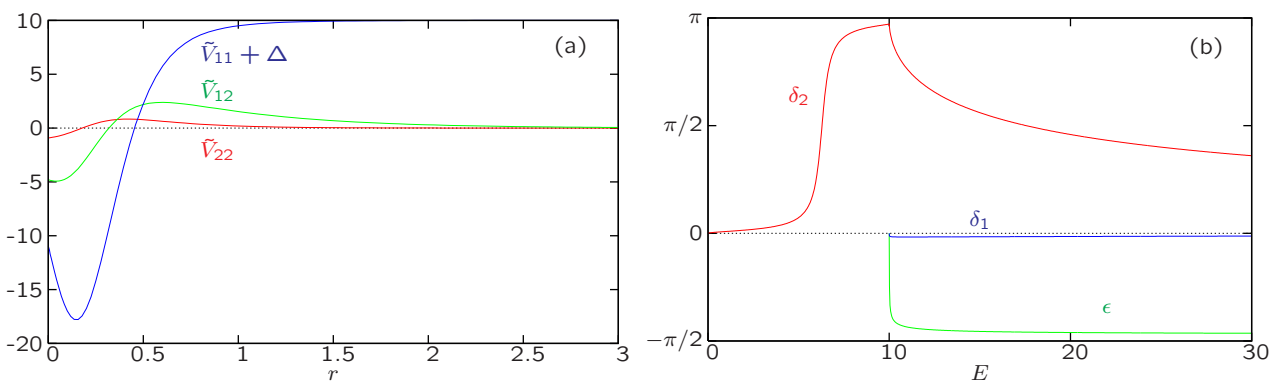

Figure 3. Same as figure 1 but for $\kappa_{2} \approx 2.194675 \ldots(\operatorname{rank} C=1)$.

\subsection{The $2 \times 2$ model with rank $C=1$}

In the previous $N=2$ example, matrix $C$ has rank 2. Let us now consider the case, not allowed in [16], where rank $C$ is 1 . Our supersymmetric formalism, on the contrary, is also valid in this case. Both matrices $Q_{0}$ and $X_{0}$ defined in Lemma 1 are numbers, $Q_{0} \equiv q_{0}, X_{0} \equiv x_{0}$, so that $Q(r) \equiv q(r)=q_{0} \exp \left(\kappa_{2} r-\kappa_{1} r\right)$ and $X(r) \equiv x(r)=x_{0} \exp \left(-2 \kappa_{1} r\right)$. Choosing $\kappa_{1}>\kappa_{2}$ and $x_{0}>-1-q_{0}^{2}$ we get from (53)

$$
U=\left(\begin{array}{cc}
a_{1} & b \\
b & a_{2}
\end{array}\right)
$$

with

$a_{1}=\frac{1-x-q^{2}}{1+x+q^{2}} \kappa_{1} \quad a_{2}=-\frac{1+x-q^{2}}{1+x+q^{2}} \kappa_{2} \quad b=\frac{2 q \sqrt{\kappa_{1} \kappa_{2}}}{1+x+q^{2}}$.

It is not difficult to check the condition rank $C=1$ (cf. (65))

$$
\left(\kappa_{1}+\alpha_{1}\right)\left(\kappa_{2}+\alpha_{2}\right)-\beta^{2}=0
$$

where $\alpha_{1,2}=a_{1,2}(0)$ and $\beta=b(0)$. We notice that our approach permits us to calculate the superpotential by the same formulas (66) and (67) as in the previous section but now the parameters are not independent anymore, they should satisfy condition (78). With the identification $x_{0}=0$ and $4 \kappa_{1} \kappa_{2} / \beta^{2}=\left(q_{0}+1 / q_{0}\right)^{2}$ this leads to an exactly-solvable model used in [11] to construct an analytical model for the Feshbach-resonance phenomenon.

Theorem 1 implies that $U(\infty)=\operatorname{diag}\left(\kappa_{1},-\kappa_{2}\right)$ which is clearly seen from (76). The value $U(0)$ is given by the same formula (76) with the replacement $x \rightarrow x_{0}$ and $q \rightarrow q_{0}$. Therefore applying Proposition 1 one gets the Jost matrix

$$
\tilde{F}(k)=\left(\begin{array}{cc}
\frac{\alpha_{1}-\mathrm{i} k_{1}}{\kappa_{1}-\mathrm{i} k_{1}} & \frac{\beta}{\kappa_{1}-\mathrm{i} k_{1}} \\
-\frac{\beta}{\kappa_{2}+\mathrm{i} k_{2}} & -\frac{\alpha_{2}-\mathrm{i} k_{2}}{\kappa_{2}+\mathrm{i} k_{2}}
\end{array}\right)
$$

which differs from (68) because of the different asymptotic form of the superpotential at infinity.

Let us now construct an explicit example that illustrates the strong impact of $U(\infty)$ on the scattering matrix. In figure 3, a potential is constructed with the same parameters as in figures 1 and 2, except for the factorization wave number which is now chosen in order to satisfy condition $(78), \kappa_{2} \approx 2.194675 \ldots$ When $\kappa_{2}$ is smaller than this limit, the potential becomes singular, a case we want to avoid here. Figures 1 (a), 2 (a) and 3 (a) show that when $\kappa_{2}$ approaches this limit value from above, the well 
in potential $\tilde{V}_{22}$ goes to infinity and finally disappears when $\kappa_{2}$ actually reaches the limit value. As long as $\kappa_{2}>2.194675 \ldots$, this well movement in $\tilde{V}_{22}$ has practically no impact on the scattering matrix: the potentials are nearly phase equivalent with each other. In contrast, when the well disappears, a strong change of behaviour is observed, as seen in figure 3 (b): while the Feshbach resonance still keeps the same energy and width, the slope of $\delta_{2}$ at zero energy becomes small and positive. Above threshold, $\delta_{2}$ has now a value very close to $\delta_{1}$ of figures 1 (b) and 2 (b), with a large negative slope. Though $\delta_{2}$ is continuous at threshold, it now has a strong cusp effect. The mixing parameter, which was close to zero, now gets close to $-\pi / 2$, while $\delta_{1}$ gets very small. The rank of $C$, and hence $U(\infty)$, thus have a strong qualitative effect on the scattering matrix, which displays very different behaviours in both cases; for practical applications, e.g. for fitting actual scattering data with such potentials, both behaviours might be of interest.

Let us finally remark that the behaviour $U(\infty)=\operatorname{diag}\left(\kappa_{1},-\kappa_{2}\right)$ agrees with the result obtained in 8 for $N=2$ in the context of a bound-state removal by supersymmetric quantum mechanics. There, the factorization matrix solution is made of one increasing and one decreasing vector solution at infinity, which corresponds to rank $C=1$. In [8], this result is even generalized to $N$ channels and a diagonal matrix for $U(\infty)$ is found, with all positive elements but one, corresponding to the channel with the lowest threshold. However, the general situation is more complicated, as described by our Theorem 2 there may be more than one negative element in the asymptotic form of the superpotential and the lowest channel may correspond to a positive asymptotic entry of the superpotential. This possibility is illustrated by our last example.

\subsection{The $3 \times 3$ model with rank $C=2$}

Here we choose $\kappa_{1}>\kappa_{3}>\kappa_{2}$, rank $C=2$ so that matrix $Q_{0}$ is the row $Q_{0}=\left(q_{0}, 0\right)$. Such a choice of $Q_{0}$ reflects the fact that the third row of $C$ is proportional to its first row (see (29)). The adopted order of thresholds corresponds to $\kappa^{\prime}=\operatorname{diag}\left(\kappa_{1}, \kappa_{2}\right)$ and $\kappa^{\prime \prime}=\kappa_{3}$. For simplicity only (equal) off-diagonal entries of $2 \times 2$ matrix $X_{0}$ from Proposition 1 are chosen different of zero which we denote $x_{0}$. Then applying (53) we obtain

$$
U=-\kappa+\frac{2}{\operatorname{det} \sigma} \kappa^{1 / 2}\left(\begin{array}{ccc}
1 & -x & q \\
-x & 1+q^{2} & -x q \\
q & -x q & q^{2}
\end{array}\right) \kappa^{1 / 2}
$$

where $\operatorname{det} \sigma=1+q^{2}-x^{2}, x=x_{0} \exp \left(-\kappa_{1} r-\kappa_{2} r\right), q=q_{0} \exp \left(\kappa_{3} r-\kappa_{1} r\right)$. The condition $\operatorname{det} \sigma \neq 0 \forall r \in[0, \infty)$ is satisfied if $q_{0}^{2}>x_{0}^{2}-1$. It is clearly seen here that at $r \rightarrow \infty$ both $q$ and $x$ vanish and the asymptotic form of $U, U_{\text {as }}=\operatorname{diag}\left(\kappa_{1}, \kappa_{2},-\kappa_{3}\right)$, agrees with Theorem 2 .

\section{Conclusion and perspectives}

In conclusion, supersymmetric quantum mechanics is a promising tool to pragmatically solve the inelastic coupled-channel inverse problem, thanks to its ability to construct sophisticated exactly-solvable models. The present work is only a starting point to the general study of supersymmetric transformations in the multichannel case: by focusing on the asymptotic behaviour of the superpotential, it reveals the richness of possible behaviours, as compared with the single-channel case. 
We have made a rigorous study of this asymptotic behaviour for a general supersymmetric transformation. Our theorem generalizes a result found in the literature [8, 9, 10]: there may be several negative elements in the asymptotic form of the superpotential and the lowest threshold may produce either a positive (as shown explicitly by our example 5.3 ) or a negative entry.

As a byproduct of our proof, we have been able to construct exactly-solvable potentials that are supersymmetric partners of the zero potential. Essential simplifications occur when each element of the factorization matrix solution is either an increasing or a decreasing exponential (not a linear combination of both types). Explicit examples have been given for two and three channels.

Future work should focus on both the behaviour of the superpotential at the origin and at infinity in the general case: arbitrary number of channels, with equal and/or different thresholds, and arbitrary rank for the matrices multiplying the regular and singular solutions in the factorization matrix solution. On the other hand, iterations of coupled-channel supersymmetric transformations should be studied, which should eventually lead to a satisfactory solution of the coupled-channel inverse problem, with and without threshold difference.

\section{Acknowledgments}

We acknowledge useful discussions with François Foucart and Andrey Pupasov, at the beginning and at the end of this work respectively. This text presents research results of the Belgian program $\mathrm{P} 5 / 07$ on interuniversity attraction poles of the Belgian Federal Science Policy Office. BFS is partially supported by grants RFBR-06-02-16719 and SS-5103.2006.2 and thanks the National Fund for Scientific Research, Belgium, for support during his stay in Brussels.

\section{References}

[1] Chadan K and Sabatier P C 1989 Inverse Problems in Quantum Scattering Theory 2nd edn (New York: Springer)

[2] Kohlhoff H and von Geramb H V 1994 Coupled channels Marchenko inversion for nucleonnucleon potentials Quantum inversion theory and applications ed von Geramb H V (Berlin: Springer) pp 315-41

[3] Cooper F, Khare A and Sukhatme U 2001 Supersymmetry in Quantum Mechanics (Singapore: World Scientific)

[4] Aref'eva I, Fernandez D J, Hussin V, Negro J, Nieto L M and Samsonov B F (eds) 2004 Progress in Supersymmetric Quantum Mechanics J. Phys. A: Math. Gen. 37 number 43 (special issue)

[5] Sukumar C V 1985 Supersymmetric quantum mechanics and the inverse scattering method $J$. Phys. A: Math. Gen. 18 2937-55

[6] Samsonov B F 1995 On the equivalence of the integral exact solution generation methods for the one dimensional Schrodinger equation to the differential ones J. Phys. A: Math. Gen. 28 6989-98

[7] Baye D and Sparenberg J-M 2004 Inverse scattering with supersymmetric quantum mechanics J. Phys. A: Math. Gen. 37 10223-49

[8] Amado R D, Cannata F and Dedonder J-P 1988 Coupled-channel supersymmetric quantum mechanics Phys. Rev. A 38 3797-800

[9] Amado R D, Cannata F and Dedonder J-P 1988 Formal scattering theory approach to $S$-matrix relations in supersymmetric quantum mechanics Phys. Rev. Lett. 61 2901-4

[10] Cannata F and Ioffe M V 1993 Coupled-channel scattering and separation of coupled differential equations by generalized Darboux transformations J. Phys. A: Math. Gen. 26 L89-92

[11] Sparenberg J-M, Samsonov B F, Foucart F and Baye D 2006 Multichannel coupling with 
supersymmetric quantum mechanics and exactly-solvable model for Feshbach resonance $J$. Phys. A: Math. Gen. 39 L639-45

[12] Samsonov B F and Shekoyan L A 1998 Investigation of a class of potentials of Bargmann type Russ. Phys. J. 41 (5) 34-9

[13] Samsonov B F and Stancu F 2003 Phase shift effective range expansion from supersymmetric quantum mechanics Phys. Rev. C $\mathbf{6 7} 054005$

[14] Taylor J R 1972 Scattering Theory: The Quantum Theory on Nonrelativistic Collisions (New York: Wiley)

[15] Newton R G 1982 Scattering Theory of Waves and Particles (New York: Springer)

[16] Cox J R 1964 Many-channel Bargmann potentials J. Math. Phys. 5 1065-9 